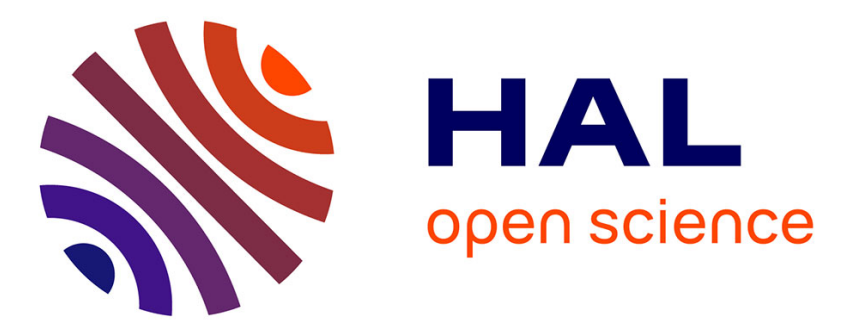

\title{
Irregular reflection of weak acoustic shock pulses on rigid boundaries: Schlieren experiments and direct numerical simulation based on a Navier-Stokes solver
}

Cyril Desjouy, Sébastien Ollivier, Olivier Marsden, Maria Karzova, Philippe Blanc-Benon

\section{To cite this version:}

Cyril Desjouy, Sébastien Ollivier, Olivier Marsden, Maria Karzova, Philippe Blanc-Benon. Irregular reflection of weak acoustic shock pulses on rigid boundaries: Schlieren experiments and direct numerical simulation based on a Navier-Stokes solver. Physics of Fluids, 2016, 28, pp.027102. 10.1063/1.4940987 . hal-01516106

\author{
HAL Id: hal-01516106 \\ https://hal.science/hal-01516106
}

Submitted on 7 Mar 2018

HAL is a multi-disciplinary open access archive for the deposit and dissemination of scientific research documents, whether they are published or not. The documents may come from teaching and research institutions in France or abroad, or from public or private research centers.
L'archive ouverte pluridisciplinaire HAL, est destinée au dépôt et à la diffusion de documents scientifiques de niveau recherche, publiés ou non, émanant des établissements d'enseignement et de recherche français ou étrangers, des laboratoires publics ou privés. 


\section{Irregular reflection of weak acoustic shock pulses on rigid boundaries : Schlieren experiments and direct numerical simulation based on a Navier- Stokes solver}

Cyril Desjouy, Sébastien Ollivier, Olivier Marsden, Maria Karzova, and Philippe Blanc-Benon

Citation: Physics of Fluids 28, 027102 (2016); doi: 10.1063/1.4940987

View online: https://doi.org/10.1063/1.4940987

View Table of Contents: http://aip.scitation.org/toc/phf/28/2

Published by the American Institute of Physics

\section{Articles you may be interested in}

Mach stem formation in reflection and focusing of weak shock acoustic pulses

The Journal of the Acoustical Society of America 137, EL436 (2015); 10.1121/1.4921681

Mach-Zehnder interferometry method for acoustic shock wave measurements in air and broadband calibration of microphones

The Journal of the Acoustical Society of America 137, 3314 (2015); 10.1121/1.4921549

Characterization of spark-generated $\mathrm{N}$-waves in air using an optical schlieren method The Journal of the Acoustical Society of America 137, 3244 (2015); 10.1121/1.4921026

Laboratory-scale experiment to study nonlinear $\mathrm{N}$-wave distortion by thermal turbulence The Journal of the Acoustical Society of America 136, 556 (2014); 10.1121/1.4887458

Random focusing of nonlinear acoustic N-waves in fully developed turbulence: Laboratory scale experiment

The Journal of the Acoustical Society of America 130, 3595 (2011); 10.1121/1.3652869

Fractal dimension computation from equal mass partitions

Chaos: An Interdisciplinary Journal of Nonlinear Science 24, 033106 (2014); 10.1063/1.4885778

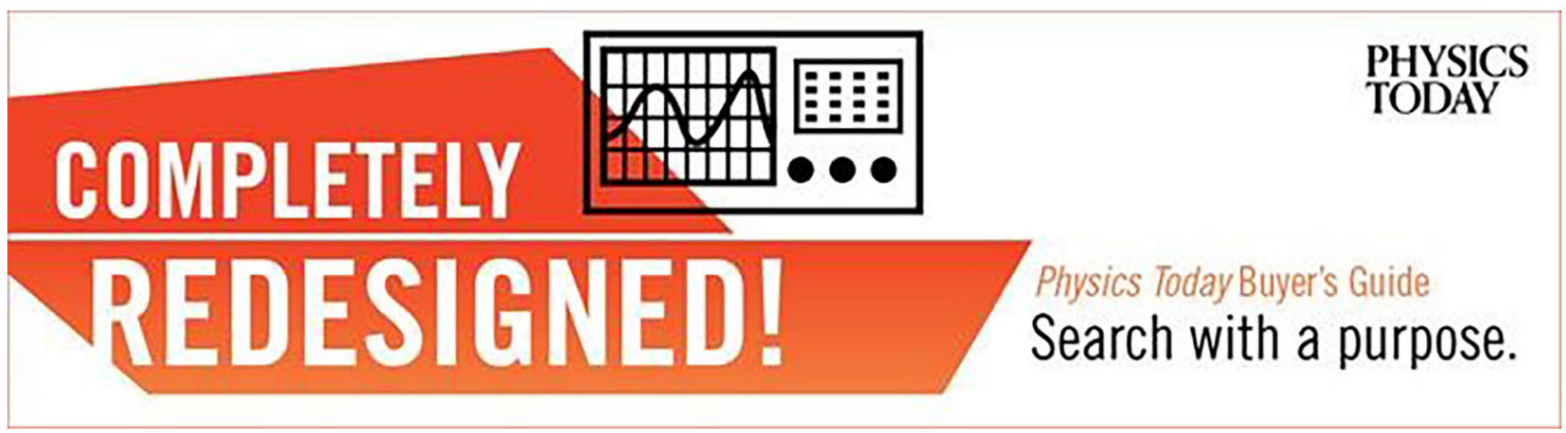




\title{
Irregular reflection of weak acoustic shock pulses on rigid boundaries : Schlieren experiments and direct numerical simulation based on a Navier-Stokes solver
}

\author{
Cyril Desjouy, ${ }^{1, a)}$ Sébastien Ollivier, ${ }^{1}$ Olivier Marsden, ${ }^{2}$ Maria Karzova, ${ }^{1,3}$ \\ and Philippe Blanc-Benon ${ }^{1}$ \\ ${ }^{1}$ Laboratoire de Mécanique des Fluides et d'Acoustique, UMR CNRS 5509, École Centrale \\ de Lyon, Université de Lyon, 69134 Ecully Cedex, France \\ ${ }^{2}$ European Center For Medium Range Weather Forecasts, Reading, United Kingdom \\ ${ }^{3}$ Department of Acoustics, Faculty of Physics, Moscow State University, Leninskie Gory, \\ Moscow 119991, Russia
}

(Received 24 June 2015; accepted 16 January 2016; published online 4 February 2016)

\begin{abstract}
The local interactions occurring between incident and reflected shock waves in the vicinity of rigid surfaces are investigated. Both regular and irregular - also called von Neumann - regimes of reflection are studied, via experimental and numerical simulations. Shock waves are produced experimentally with a $20 \mathrm{kV}$ electrical spark source which allows the generation of spherically diverging acoustic shocks. The behaviour of the resulting weak acoustic shocks near rigid boundaries is visualized with a Schlieren optical technique which allows the spatial structure of the shocks to be studied. In particular, the evolution of the Mach stem forming above a flat surface is examined, and its height is observed to be directly linked to the angle of incidence and the pressure amplitude of the incident shock. The propagation of an acoustic shock between two parallel rigid boundaries is also studied. It is shown that the strong interactions between the Mach stems emerging from the two boundaries can lead to a drastic modification of the morphology of the acoustic field in the waveguide. Experimental results are compared to numerical results obtained from high-order finite-difference based simulations of the 2D Navier-Stokes equations. The good agreement between the experimental distribution of the acoustic field and numerical results suggests that numerical simulations are promising as a predictive tool to study nonlinear acoustic propagation of acoustic waves in complex geometrical configurations with rigid boundaries. (C) 2016 AIP Publishing LLC. [http://dx.doi.org/10.1063/1.4940987]
\end{abstract}

\section{INTRODUCTION}

Ernst Mach discovered experimentally in $1878^{1}$ that the point of intersection of a strong incident shock and its reflection from a rigid boundary can detach from this boundary under particular conditions. This phenomenon leads to the formation of a third shock, called Mach stem, that ensures the connection between the intersection of incident and reflected waves and the rigid boundary. This kind of reflection is called irregular reflection or Mach reflection in honor of its discoverer. Since then, an entire field of research has focused on the study of shock-wave reflection phenomena. ${ }^{2}$

In particular, in 1943, John von Neumann studied theoretically the experimental observations of Ernst Mach and introduced a new theory, called three-shock theory, ${ }^{3}$ contrasting with the classical two-shock theory which is valid as long as the intersection of incident and reflected shocks remains on the boundary (case of regular reflection). A number of theoretical, numerical, and experimental studies ensued from these works. It has been shown that there is a good agreement between

\footnotetext{
a) cyril.desjouy@ gmail.com
} 
TABLE I. Regimes of reflection as a function of the critical parameter $a$ observed numerically in the case of step shock. ${ }^{7}$

\begin{tabular}{llc}
\hline \hline Kind of reflection & \multicolumn{1}{c}{ Regime } & Step shock \\
\hline $\begin{array}{l}\text { Classical Snell-Descartes } \\
\text { reflection }\end{array}$ & Linear regular regime & $a>5$ \\
Generalized Snell-Descartes & Nonlinear regular & $\sqrt{2}<a<5$ \\
reflection & regime & \\
von Neumann reflection & Irregular regime & $0.4<a<\sqrt{2}$ \\
Weak von Neumann reflection & Irregular regime & $a<0.4$ \\
\hline \hline
\end{tabular}

the von Neumann's three-shock theory and experiments for strong shocks (Mach number $M \gg 1$ ). ${ }^{4}$ In the case of weak shock reflection $(M \simeq 1)$, three-shock theory does not predict Mach reflection while experiments and numerical simulations have shown that irregular reflections can still exist. ${ }^{4,5}$ This discordance is called the von Neumann paradox.

In the field of acoustics for which $M-1$ is of order of $10^{-4}$, this paradox was observed numerically by Sparrow and Raspet ${ }^{6}$ when they investigated the reflection from a rigid boundary of a spherical spark generated acoustic shock. This paradox was later studied numerically by Baskar et $a .^{7}$ In particular, they investigated the phenomenon of transition between regular and irregular regimes of reflection using the $\mathrm{KZ}$ equation ${ }^{8}$ in the case of step shocks, plane $N$-waves, and of periodic sawtooth waves. The derivation of the boundary conditions for the KZ equation leads them to introduce a critical parameter $a$ describing the ratio of nonlinear and diffraction effects near the rigid boundary. This parameter is defined as

$$
a=\frac{\sin \theta_{i}}{\sqrt{2 \beta P_{\max } /\left(\rho c^{2}\right)}},
$$

with $\theta_{i}$, the angle of incidence of the shock wave, $P_{\max }$, the positive peak pressure amplitude of the incident shock, $\beta$, the nonlinear parameter, $\rho$, the density of fluid, and $c$, the adiabatic celerity of sound. As described in Table I, four regimes have been defined for step shocks as a function of this parameter. When $a$ is larger than 5, the classical Snell-Descartes reflection takes place. The regime is linear regular: the angles of incidence and reflection are equal. When this parameter lies between $\sqrt{2}$ and 5, the regime of reflection become nonlinear regular. In this case a generalized Snell-Descartes reflection is observed: the angles of incidence and reflection can be different. The transition between regular and irregular regimes is given theoretically by $a=\sqrt{2}$. ${ }^{7}$ Below this value, regular reflection cannot exist anymore. It is worth noting that this limit has also been obtained by Brio and Hunter ${ }^{9}$ from the two-dimensional Burgers equation in the case of step shocks. For values of $a$ between 0.4 and $\sqrt{2}$, the incident and reflected shocks intersect above the rigid boundary, giving rise to the Mach stem. The intersection between these three shocks is called the triple point $\mathrm{T}$ [see Fig. 1], and this regime of reflection is called von Neumann reflection in the case of weak acoustic shocks. Finally, when the critical parameter $a$ is lower than 0.4 , a new regime of reflection takes place: the reflected shock tends to disappear as the angle of incidence approaches the perfectly grazing angle. This phenomenon is introduced by Baskar et al. as weak von Neumann reflection. ${ }^{7}$ In the case of $N$-waves and periodic sawtooth waves, Baskar et al. reported a similar categorization.

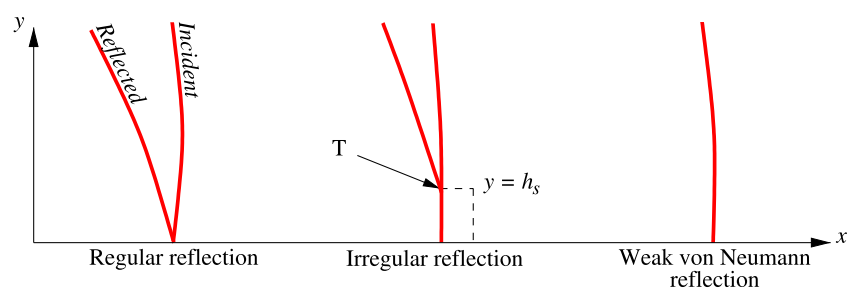

FIG. 1. Regimes of reflection reported by Baskar et al. in the case of step shocks. ${ }^{7}$ 
Those different regimes have been observed experimentally for plane ultrasonic shock waves in water by Marchiano et al. ${ }^{10}$ They reported a good agreement between the numerical and experimental transitions from Snell-Descartes reflection to von Neumann reflection, and from the latter, to weak von Neumann reflection. The fact is that acoustic shocks are not just limited to plane waves. They can be focused ultrasonic shock waves which are widely used in ultrasound therapy, sonic booms produced by aircraft, or Mach waves produced by supersonic projectiles, or blasts. For the latter case, the shock wave has a spherical morphology and diverges spherically. As previously mentioned, Sparrow and Raspet ${ }^{6}$ observed numerically the formation of the Mach stem during the propagation of incident and reflected spherically diverging shock waves produced by a spark source. Recently, Karzova et al. also reported experimentally (from schlieren measurements using a single mirror coincident system) the arising of Mach stem from the reflection of a spherically diverging $N$-wave on a rigid boundary for small angles of incidence. ${ }^{11,12}$ They found a categorization close to that presented in Table I for the different regimes of reflection. In particular, they reported a transition between regular and irregular regimes of reflection for $a \simeq 1.1 \pm 0.3$ in the case of spherically diverging shocks, which is close to the value reported by Baskar et al. in the case of step shocks.

The study of acoustic shock propagation in complex environments is of great interest to several areas of acoustics. Whether it be for medical applications such as ultrasound therapy, in aviation where the sonic boom phenomenon is widely investigated, or for military applications involving supersonic projectiles or explosions, the comprehension of shock wave propagation is essential, and particularly so for spherically diverging acoustic shocks. However, to our knowledge, few studies have focused on the propagation and reflection phenomena of spherical weak acoustic shock waves. Such waves, usually generated by a spark source, are widely used, particularly in urban acoustics ${ }^{13}$ and laboratory scaled experiments. ${ }^{14,15}$ Hence, the aim of this paper is to study, both experimentally and numerically, the reflection of a spherically diverging weak acoustic shock wave produced by an electrical spark source and to complete previous experimental and numerical observations. ${ }^{7,11}$ In particular, the experimental works of Karzova have been extended to a wider range of experimental configurations, and an in-depth study of the evolution of the Mach stem is achieved using an optical schlieren method. The images obtained from this method provide access to the morphology of the acoustic shocks, and when it exists, to the reflection pattern. Experimental results are compared to numerical simulations based on high-order finite difference solutions of the two-dimensional Navier-Stokes equations, and a parametric study is undertaken to characterize the evolution of the Mach stem generated by the propagation of cylindrical acoustic shocks. The propagation and reflection of spherical shock waves between two parallel rigid boundaries constitute a final original point of interest.

Experimental and numerical setups are presented in Sections II and III, respectively. Then, the reflection of a spherically diverging shock wave is investigated. Spatial distributions of acoustic pressure obtained numerically are compared to schlieren measurements in Section IV. Particular attention is paid to the characterization of the Mach stem evolution. Section V is devoted to the study of the propagation of a spherically diverging acoustic shock between two parallel rigid boundaries, and particularly to the interactions between the Mach stems arising from each of the two boundaries.

\section{EXPERIMENTAL SETUP}

A diagram of the experimental setup is shown in Fig. 2. A $20 \mathrm{kV}$ electrical spark source is used to generate high amplitude pressure pulses in air. With a gap between the tungsten electrodes adjusted to $20 \mathrm{~mm}$, the generated spark gives rise to an acoustic blast wave which diverges spherically in the measurement domain. The spatial distribution of the acoustic field is measured using a Z-type schlieren system. ${ }^{16-18}$ A continuous quartz tungsten halogen white light source (maximum $250 \mathrm{~W}$ ) creates a light beam between two parallel parabolic mirrors (108 $\mathrm{mm}$ diameter, $864 \mathrm{~mm}$ focal length) separated by approximately $1.7 \mathrm{~m}$. Optical aberrations are reduced by limiting the off-axis to $2 \alpha<10^{\circ}$. A spatial filtering is operated with a knife edge located in front of the CMOS 


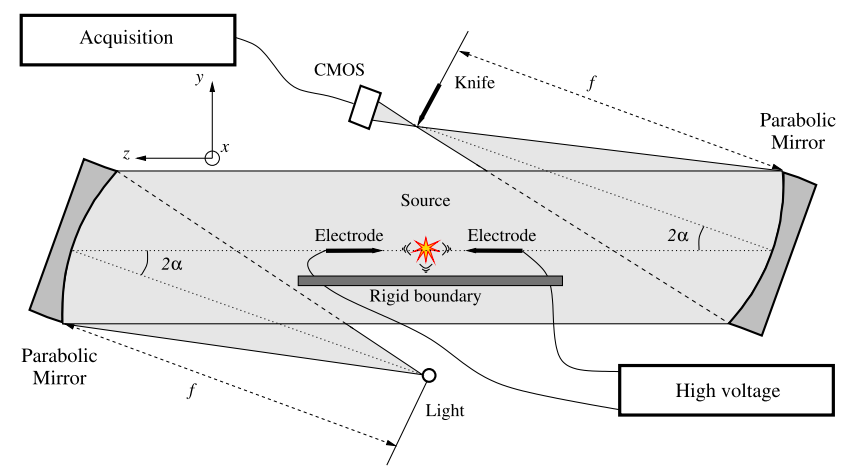

FIG. 2. Schematic representation of the Z-type schlieren system. A light beam is created between two parabolic mirrors. The propagation of a shock through this beam leads to variations of the light intensity which are linked to the variations of acoustic pressure.

camera (Phantom V12), at a distance equal to the focal length $f$, perpendicularly to the direction of propagation of the shock wave. Schlieren images are recorded with a resolution of $800 \times 600$ pixels, a frame rate of $18 \mathrm{kHz}$ and an exposure time of $1 \mu \mathrm{s}$. They represent the variations of light intensity in the measurement domain which are linked to the variations of density $\rho$, and consequently of acoustic pressure $p_{a}{ }^{16}$

Schlieren images are analyzed in order to extract the geometrical parameters of the shocks. First, an averaged background image is calculated for each set of measurements and is subtracted from images including the shocks. Then, a marching squares algorithm is applied to locate the shock positions. This algorithm is a particular case of the marching cubes algorithm. ${ }^{19}$ The incident and reflected shocks are approximated far from the boundary by a least-squares method (under the assumption of a locally spherical wavefront) to determine the locations of the source, the source image, the triple point, and the angles of incidence and reflection [see Fig. 1]. Due to the resolution of the schlieren images (one pixel represents $0.17 \mathrm{~mm}$ ) and the exposure time of $1 \mu \mathrm{s}$ (during which the wave propagates over around $0.34 \mathrm{~mm}$ ), the minimum uncertainty on the determination of the spatial characteristics of the acoustic field is of about $0.51 \mathrm{~mm}$. Moreover, even if the measurements present an acceptable signal-to-noise ratio (SNR of about $17 \mathrm{~dB}$ ), in some image areas, the lack of contrast can lead to additional uncertainties. In particular, in the vicinity of the triple point, there may be strong interactions between the reflected shock front and the incident rear shock leading to uncertainties on the determination of the height $h_{s}$ of the triple point that can reach $2 \mathrm{~mm}$.

Therefore, schlieren photography enables the investigation of the morphology of the acoustic pressure in the measurement domain. However, this technique does not provide direct access to the acoustic pressure amplitude. Simultaneous measurements with classical condenser microphones being inadequate considering the pressure magnitudes and frequency ranges close to the spark source, preliminary characterization of the spark source has been carried out in free field conditions using a Mach-Zehnder interferometer. ${ }^{20,21}$ These preliminary results have been used to determine the pressure magnitude ${ }^{22-24}$ in the experimental spatial measurement domain considered in this paper, i.e., for $x \in[40,380] \mathrm{mm}$. Over this spatial range, the propagation distance, the wavelength $\lambda$, and amplitude $P_{\max }$ of the generated shock lie between $\left[1 \times 10^{-2}, 2 \times 10^{-2}\right] \mathrm{m}$, and $[0.6,8] \mathrm{kPa}$, respectively. For given atmospheric conditions, the acoustic shocks generated by the spark source are highly reproducible (maximum variations of amplitude and wavelength of about 2\%). Preliminary schlieren measurements have also been conducted in free field conditions to ensure that the acoustic shock generated by the spark source is spherical.

Experiments have been carried out for several geometrical configurations. As Fig. 3 shows, the angle of incidence $\theta_{i}$ can be adjusted by changing the height $y_{s}$ of the spark source and/or the center of the observation window located at $x=x_{0}$. Experiments have been done for angles of incidence $\theta_{i}$ from $1^{\circ}$ to $18^{\circ}$ and positive peak pressure amplitude $P_{\max }$ of the incident shock from 0.6 to $8 \mathrm{kPa}$. In these configurations, the values of the critical parameter $a$ range from 0.05 to 3 . 


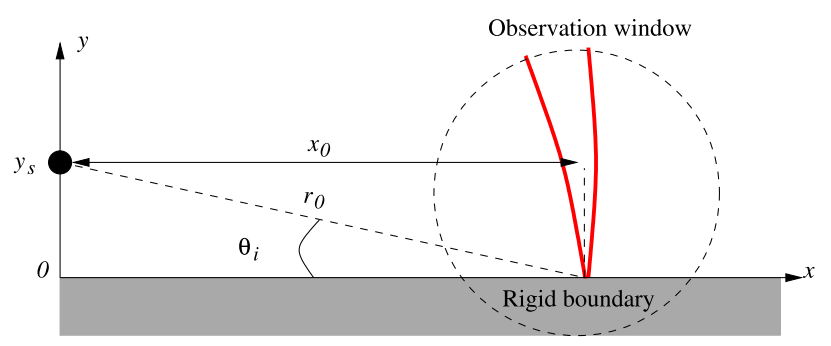

FIG. 3. Schematic representation of the geometrical setup. A spherically diverging shock wave generated by the spark source located at $\left(0, y_{s}\right)$ propagates and passes through the observation window whose center is located at the distance $x_{0}$ from the spark source.

\section{NUMERICAL METHOD}

The full two-dimensional Navier-Stokes equations are solved to model the propagation and the reflection of weak shock waves. The description of the solver is detailed in Ref. 25 and summarized hereafter. Explicit finite differences based on 11-point stencils are used to compute the spatial derivatives involved in the Navier-Stokes equations. ${ }^{26}$ Time integration is performed with a six-stage optimized Runge-Kutta scheme. ${ }^{26}$ Additionally, a shock-capturing filtering technique is employed in order to handle the discontinuities that appear in the vicinity of shock waves. The shock-capturing methodology employed in this work is based on the application of a low-order (second order) filter to flow variables in the vicinity of acoustic shocks, in addition to the high-order selective filtering which is applied over the entire computational domain. Acoustic shocks are identified by comparing the level of high-frequency pressure fluctuations at each grid point to the local mean ambient pressure. High values of this ratio are indicative of a shock front. At each time step, the second order filter is applied with a local strength dependent on the local value of the ratio: low values lead to a filtering magnitude of zero, while values above a certain threshold ${ }^{27}$ generate a positive filtering magnitude. Thus, away from acoustic shocks, flow variables are unchanged by the shock-capturing scheme, while near shocks, high frequency energy is damped, leading to smoother shock fronts, of the order of four grid points in thickness, and free from significant Gibbs oscillations. A detailed description of the methodology can be found in Bogey et al. ${ }^{27}$ This paper briefly describes different shock-capturing techniques found in the computational fluid dynamics literature, as well as their typical deficiencies for acoustic computations. The paper then provides a detailed spectral analysis of the adaptive filtering procedure, before examining the effects of the methodology on several linear and nonlinear test cases. ${ }^{27}$ The general flow features, including shock front locations, are preserved, while Gibbs oscillations are strongly reduced.

The Navier-Stokes solver has been fully implemented in OpenCL. This solver does not account for molecular relaxation effects which contribute to acoustic absorption and dissipation during the propagation process. Even if these dissipative and dispersive effects can be taken into account, ${ }^{28}$ it has been shown that, in the case of the propagation of acoustic shocks over short distances (in the present case, of about ten wavelengths), they are negligible compared to nonlinear and absorption effects. ${ }^{29}$

The boundary condition on the rigid wall is a non-slip condition. In the following, numerical acoustic pressure distributions are presented for $4096 \times 2048$ grid meshes, with spatial and time steps of $0.1 \mathrm{~mm}$ and $1.5 \times 10^{-5} \mathrm{~s}$, respectively. The source is a Gaussian-envelope injection of energy. The adjustment of its amplitude and half-width allows a wide range of acoustic waveforms to be established in the computation domain.

The comparison between numerical and experimental results is achieved by adjusting the initial wavelength and peak amplitude of the source in order to obtain a numerical wave that fits the experimental waveform in the free field at a distance $x \simeq x_{0}$ (i.e., at the center of the observation window). Examples of time waveforms obtained numerically and experimentally in the free field are presented in Fig. 4. Weak Gibbs oscillations are visible on the numerical shock front, but the experimental and numerical waveforms are close, in terms of rise time, duration, and also positive 


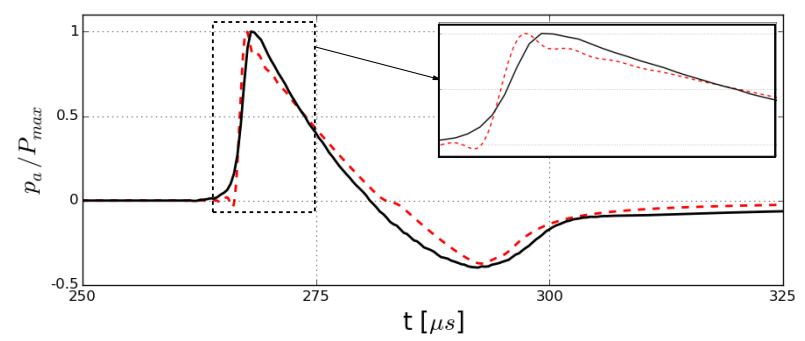

FIG. 4. Example of experimental (solid line) and numerical normalized (dashed line) waveforms in the free field at a propagation distance of $x=90 \mathrm{~mm}$ from the source.

and negative peak pressures. It is worth noting that this work focuses on the study of the spatial distribution of the acoustic field, not on the temporal waveforms. The aforementioned oscillations could incidentally be reduced by using shorter temporal and spatial steps but at the cost of a significantly longer computation time. The numerical parameters used in the present work (temporal and spatial steps) are a compromise allowing a parametric study for thousands of geometrical cases to be carried out in a reasonable time.

\section{REFLECTION ON A RIGID BOUNDARY}

\section{A. Regimes of reflection}

As described above, different regimes can take place when an acoustic shock is reflected from a rigid boundary. Fig. 5 presents results of numerical simulations for different values of the critical parameter $a$ of the normalized acoustic pressure $p_{a} / P_{\max }, P_{\max }$ being the positive peak pressure amplitude. When $a$ is greater than $\sqrt{2}$ [Fig. 5(a)], the regime of reflection is regular. Incident and reflected shocks intersect on the rigid boundary and angles of incidence and of reflection are equal. Increasing the incident pressure amplitude and/or decreasing the angle of incidence makes the parameter $a$ decrease. When $a$ becomes lower than the critical value $a=\sqrt{2}$ [Fig. 5(b)], the
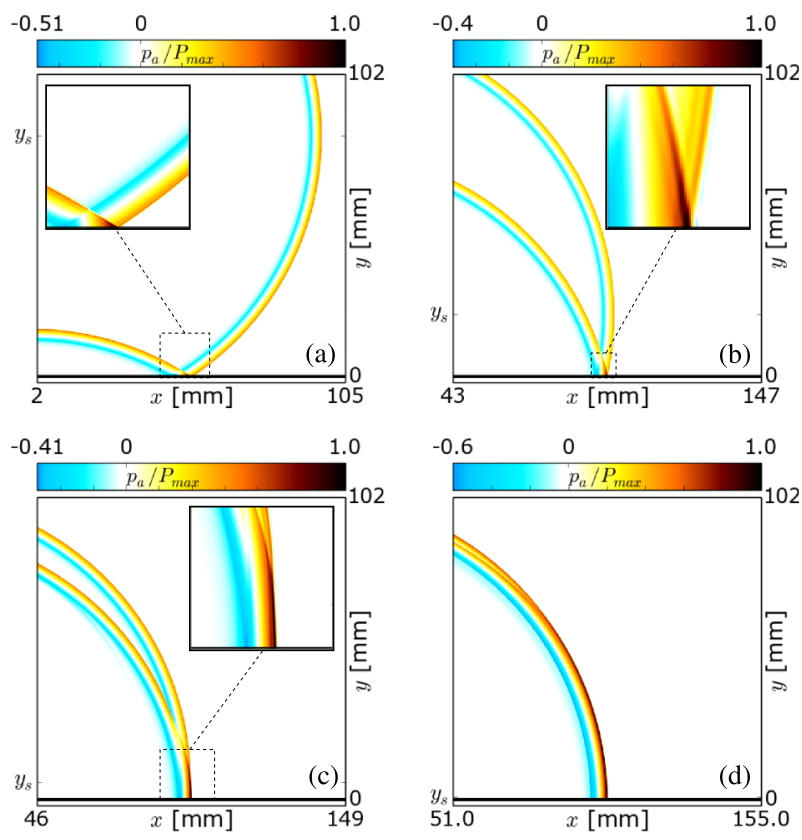

FIG. 5. Normalized numerical distribution of acoustic pressure $p_{a} / P_{\max }$ for: (a) $a=5.01$, (b) $a=1.31$, (c) $a=0.37$, (d) $a=0.06$. A zoom on the intersection of incident and reflected shocks is also presented in the boxes inserted in (a)-(c). 
regime of reflection evolves from regular to irregular. Then, the angles of incidence and reflection are noticeably different, and the intersection of incident and reflected shocks starts to move away from the rigid boundary, leading to the formation of the Mach stem. In Fig. 5, the height $h_{s}$ of the Mach stem evolves from $h_{s}=2 \mathrm{~mm}$ [Fig. 5(b)] to $12 \mathrm{~mm}$ [Fig. 5(c)]. As $a$ decreases more, the intersection of incident and reflected shocks continues to moves away from the boundary and then, the reflected shock tends to merge gradually with the incident shock. When the angle of incidence approaches the perfectly grazing angle ( $a$ tends to 0 ), the reflection tends to a weak von Neumann reflection as shown in Fig. 5(d).

Fig. 6 presents the normalized maximum positive peak pressure $P_{\max } / P_{\infty}$ on the rigid boundary obtained from the two-dimensional simulations as a function of the parameter $a, P_{\infty}$ being the positive peak pressure for $a=3.5$. This normalization aims at obtaining an amplification of 2 for large values of $a$ in order to represent the doubling of the incident pressure for large angles of incidence, i.e., for a regular regime of reflection, in accordance with the linear Snell-Descartes laws. As the parameter $a$ decreases, the maximum pressure increases to a maximum amplification of about 2.4 for $a=1.1$, i.e., for an irregular regime of reflection. It is worth noting that the non-constant amplification exhibited for $a>\sqrt{2}$ is predicted by the two-shock theory. As $a$ decreases further, the maximum amplification decreases below 2 around $a=0.5$. Then, the amplitude continues to decrease to 1.1 for $a=0$, i.e., for the perfect grazing incidence. At the same time, the regime of reflection changed from a 2 -shocks regime (classical doubling of the pressure) to a 3-shocks regime (continuous increase, then decrease of the amplification around the critical value $a=\sqrt{2}$ ), and finally to a 1-shock regime (no longer a doubling of pressure). The transition between the different regimes of reflection is continuous as reported by Baskar et al. ${ }^{7}$ in the case of step shocks.

These results are close to the ones obtained previously in the literature. ${ }^{7,11}$ The categorization reported by Baskar et al. [see Table I] remains valid except for the nonlinear regular regime. Indeed, in the case of a cylindrical acoustic shock, the angle of reflection always matches the angle of incidence for critical parameters $a>\sqrt{2}$, so that above this critical value, the regime of reflection is governed by the classical Snell-Descartes laws. It is also worth noting that weak von Neumann reflection occurs for values of $a<0.1$ in the case of cylindrical shocks versus values of $a<0.4$ in the case of step shocks.

Comparisons with experimental measurements have been done in the case of irregular reflections. Fig. 7 reports numerical normalized distributions of acoustic pressure (top figures) and corresponding schlieren snapshots (middle figures) for different $a$ values lower than $\sqrt{2}\left(y_{s}=6\right.$, 11,16 , and $21 \mathrm{~mm}$, for $x_{0} \simeq 100 \mathrm{~mm}$ ), so that the regime of reflection is irregular. There is an excellent correlation between experimental and numerical results, whether it is for the front shock, the rear shock, or the Mach stem shape and location. To illustrate this, the bottom plot of Fig. 7 shows the comparison between the outlined front shocks (incident, reflected, and Mach stem) seen numerically and experimentally. Only small differences are observed on the shock patterns at the top of the observation window, where the calculated and the measured shock locations differ only by about one millimeter. The main source of uncertainty in the experiments comes from the random fluctuations of the spark location, which result in an error of the same order on the estimation of the propagation distance. The difference between the three-dimensional (experiments) and the

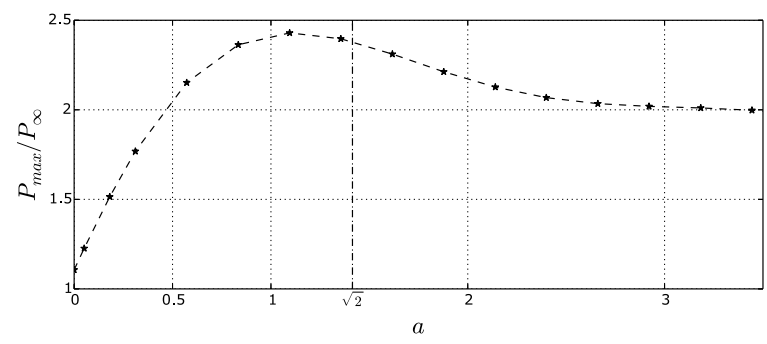

FIG. 6. Normalized maximum positive peak pressure $P_{\max } / P_{\infty}$ on the rigid boundary as a function of the critical parameter a. $P_{\infty}$ is the maximum positive peak pressure for $a=3.5$. 

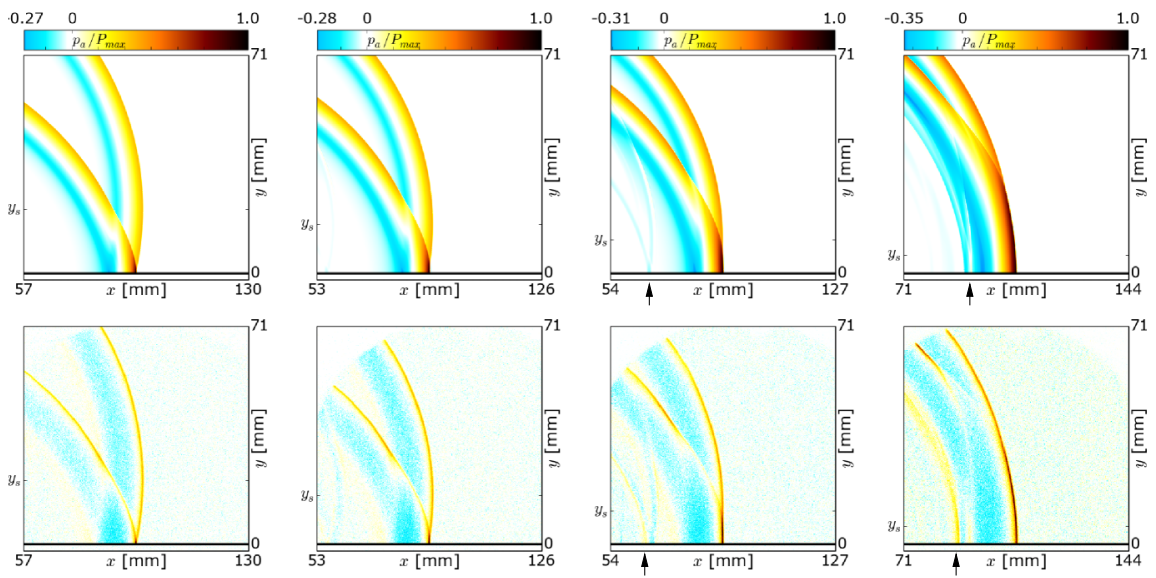

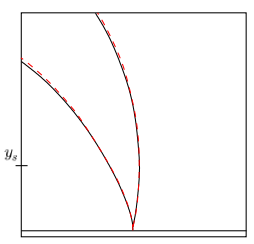

(a)

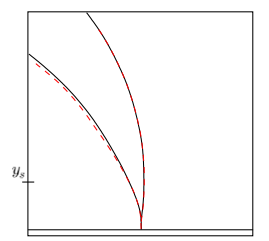

(b)

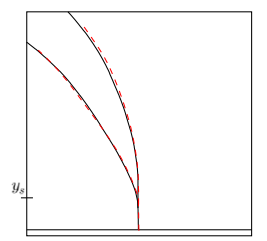

(c)

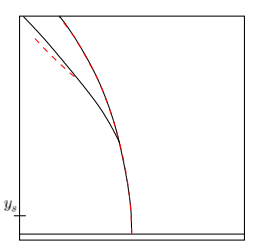

(d)

FIG. 7. Comparisons of numerical normalized spatial distribution of the acoustic pressure $p_{a} / P_{\max }$ (top figures) and schlieren images (middle figures) as a function of the critical parameter: (a) $y_{s}=21 \mathrm{~mm}, a=0.83$, (b) $y_{s}=16 \mathrm{~mm}, a=0.66$, (c) $y_{s}=11 \mathrm{~mm}, a=0.45$, and (d) $y_{s}=6 \mathrm{~mm}, a=0.22$. The bottom figures present a comparison of the front shocks outlined from the numerical (solid black lines) and schlieren (dashed red lines) results.

two-dimensional propagation (simulations) may also contribute to additional discrepancies between the measured and the calculated patterns.

As shown in Fig. 7, when the critical parameter $a$ decreases, the location of the triple point gradually moves away from the rigid boundary, so that the height of the Mach stem $h_{s}$ increases as the parameter $a$ decreases : $h_{s}=3 \mathrm{~mm}, 4.5 \mathrm{~mm}$, and $9 \mathrm{~mm}$ for $a=0.88,0.66$, and 0.45 , respectively. As long as $a$ is greater than approximately 0.41 [Figs. 7(a)-7(c)], the shape of the Mach stem is a straight line. Obviously, this causes a local loss of circularity of the wavefront in the vicinity of the Mach stem. This deformation is due to the positive peak pressure amplitude of the Mach stem which is higher than the amplitudes of both incident and reflected waves, leading to the fact that the Mach stem propagates faster than the incident and reflected waves. This increase of the speed of the Mach stem contributes to counterbalance the curvature of the circular wavefront near the rigid boundary. When $a$ is lower than 0.41 [Fig. 7(d)], the location of the triple point is higher than the source location $\left(y_{s}=6 \mathrm{~mm}, h_{s}=29 \mathrm{~mm}\right)$. The differences between the peak positive pressure of the Mach stem and the incident shock are in that case not enough to counterbalance the differences in speeds of propagation. Accordingly, the Mach stem becomes curved, and the shape of the wavefront (which includes the incident and Mach waves) is elliptic.

It is worth noting that a secondary pattern propagates behind the incident and reflected shocks, at a distance equal to twice the height of the source $y_{s}$. The location of this secondary pattern is pointed out on Figs. 7(c) and 7(d) (arrow on the $x$-axis). It has been identified as a reflection at the source location. The sudden increase of pressure at the spark source location goes along with a locally large increase in temperature (several hundred Kelvin). Since the characteristic time of establishment of a homogeneous distribution of temperature in the vicinity of the spark source is several orders of magnitude larger than the time of propagation from the source to the boundary, there still exist a strong temperature gradient at the spark source location when the reflected shock propagates into this area. Then the reflected shock is diffracted when it hits this temperature spot and this diffraction gives rise to new incident and reflected shocks which are observed both experimentally and numerically. 


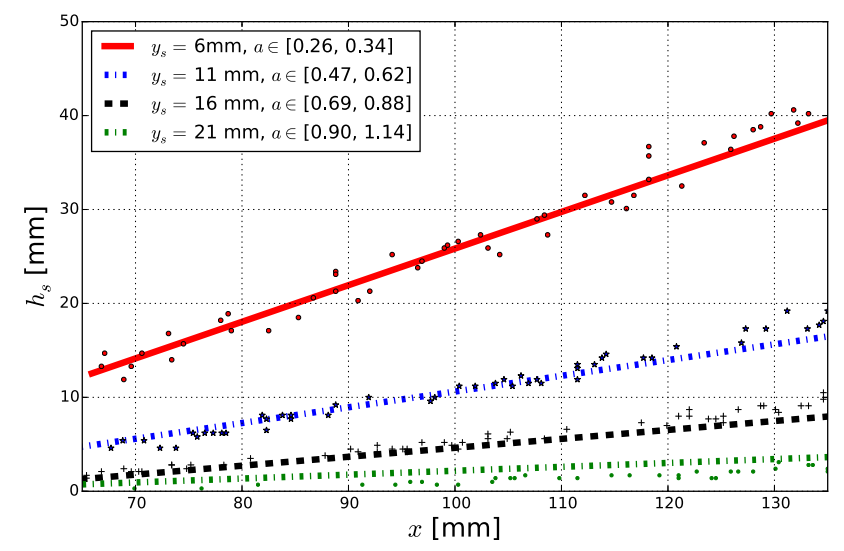

FIG. 8. Comparison of the numerical (lines) and experimental (markers) evolutions of the Mach stem height $h_{s}$ as a function of the propagation distance $x$ for four positions of the acoustic source : $y_{s}=6,11,16,21 \mathrm{~mm}$.

\section{B. Evolution of the Mach stem}

As was shown, the height $h_{s}$ of the Mach stem changes as a function of the critical parameter $a$ as long as the regime of reflection is irregular. Fig. 8 presents numerical (lines) and experimental (points) evolution of the height $h_{s}$ of the Mach stem as a function of the distance of propagation, $x$, from approximately 70 to $130 \mathrm{~mm}$, for four positions of the source, namely, $y_{s}=6,11,16$, and $21 \mathrm{~mm}$. In these geometrical configurations, and as a function of the corresponding peak pressure amplitudes, the parameter $a$ lies between 0.26 and 1.14 , so that the regime of reflection is always irregular. It is worth noting that since the peak pressure amplitude evolves with respect to the propagation distance, there are ranges of $a$ values corresponding to each of the four cases presented in Fig. 8. The Mach stem height $h_{s}$ evolves in a linear way as a function of the distance of propagation $x$ for the four cases. For the lower values of parameter $a\left(y_{s}=6 \mathrm{~mm}\right)$, the height $h_{s}$ of the Mach stem lies between 12 and $40 \mathrm{~mm}$ for the propagation distances considered here. Experimental results deviate slightly from the numerical ones (up to $7.5 \%$ ), probably due to the uncertainty on the determination of the experimental height $h_{s}$ (of about $2 \mathrm{~mm}$ ). When the parameter $a$ increases, the height of the Mach stem decreases. This leads necessarily to a larger deviation. Indeed, in the case where $y_{s}=11 \mathrm{~mm}$, the height $h_{s}$ of the Mach stem lies between 5 and $16 \mathrm{~mm}$ with a deviation of up to $9 \%$. When $y_{s}=16 \mathrm{~mm}, h_{s}$ ranges from 1 to $8 \mathrm{~mm}$ with a maximal deviation of $22 \%$. Finally, when $y_{s}=21 \mathrm{~mm}, h_{s}$ lies between 1 and $4 \mathrm{~mm}$ with a maximal deviation of $55 \%$. In these two latter cases, the Mach stem is barely formed, and the order of magnitude of its height $h_{s}$ is close to the measurement uncertainty. A comparison between numerical and experimental results is also achieved considering the linear regression of the experimental results. For $y_{s}=6,11,16$, and $21 \mathrm{~mm}$, the associated experimental and numerical slopes are $0.40,0.20,0.11,0.03$, and $0.39,0.17$, $0.10,0.04$, respectively. The numerical and experimental evolutions of the height of the Mach stem are in good agreement.

To characterize more precisely the evolution of the Mach stem, a parametric numerical study has been conducted over about 2000 configurations (different sets of source locations, propagation distances, and incident acoustic pressures). Figs. 9(a) and 9(b) present a summary of this study through the linear and logarithmic evolutions of the normalized height $h_{s} / y_{s}$ of the Mach stem, respectively, as a function of the critical parameter $a$. These results show a weak dispersion of $h_{s} / y_{s}$ values due to the uncertainty in the numerical determination of the height $h_{s}$. This uncertainty mainly comes from the numerical spatial step (i.e., $0.1 \mathrm{~mm}$ ). Hence, as long as $h_{s}$ is greater than $10 \mathrm{~mm}$, the error in its determination is lower than $1 \%$. When $h_{s}$ is of the order one millimeter, the error in its determination can reach $10 \%$. Nevertheless, Fig. 9(b) highlights a clear relationship between the ratio $h_{s} / y_{s}$ and the critical parameter $a$ that can be written as

$$
\frac{h_{s}}{y_{s}}=\left(\frac{\xi}{a}\right)^{2},
$$



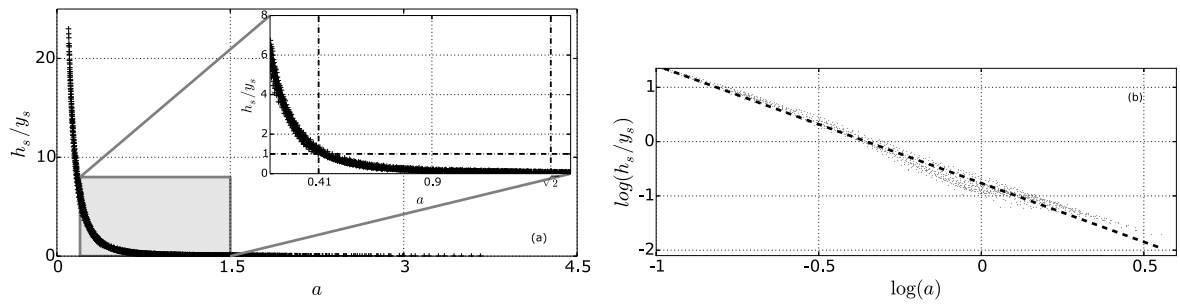

FIG. 9. Evolution of the normalized Mach stem height $h_{s} / y_{s}$ as a function of the critical parameter $a$, (a) in linear scale, and (b) in logarithmic scale.

$\xi$ being a constant. Approximating the numerical results presented in Fig. 9(b) with Eq. (2) leads to $\xi=0.41$. Note that the meaning of this value remains to be established.

As expected, Fig. 9(a) shows that the ratio $h_{s} / y_{s}$ remains nominally zero as long as $a>\sqrt{2}$, so that the regime of reflection is regular, and there is no Mach stem. Then, when the parameter $a$ decreases below the critical value $\sqrt{2}$, the ratio $h_{s} / y_{s}$ increases and the Mach stem starts to emerge. The height of the Mach stem $h_{s}$ increases as the parameter $a$ decreases until the singular value $h_{s} / y_{s}=1$ for $a=0.41$, for which the location of the triple point matches the location $y_{s}$ of the source. As long as $a$ is lower than 0.41 , the height $h_{s}$ of the Mach stem exceeds the location of the source $y_{s}$. When the parameter $a$ is lower than 0.1 , the reflected shock almost disappears. In this situation, the location of the triple point is no longer defined, likewise for the height $h_{s}$ of the Mach stem. In addition to the previous results, the ratio $h_{s} / y_{s}$ obtained numerically is presented in Fig. 10 as a function of the positive peak pressure amplitude $P_{\max }$ obtained from the two-dimensional simulations and the angle of incidence $\theta_{i}$. This map shows that the transition between regimes of reflection is more sensitive to the angle of incidence than to the positive peak pressure amplitude. Indeed, at a given pressure, decreasing the angle of incidence quickly leads to irregular regimes whereas, at a given angle of incidence, increasing the peak pressure amplitudes is not always enough to lead to irregular regimes. In the domain considered here, variations of about $10 \%$ of the angle of incidence or of the positive peak pressure amplitude leads to variations of about $10 \%$ or $5 \%$, respectively, on the value of the parameter $a$. This is due to the transition between regular and irregular regimes that is mostly ruled by $\sin \theta_{i}$ (See Eq. (1)). It should also be mentioned that this higher dependence on the angle of reflection can explain the good agreement between the experiments and the two-dimensional model. Indeed, given that the numerical pressure amplitude is adjusted to be equal to the experimental one at a distance from the source $x=x_{0}$, the differences between the two and three-dimensional variations of the pressure amplitude during the propagation of the acoustic shock over several wavelengths (less than ten) is not sufficient to lead to drastic modifications of the morphology of the acoustic field, and in particular of the Mach stem behavior. Significant differences would undoubtedly appear if larger propagation distances were

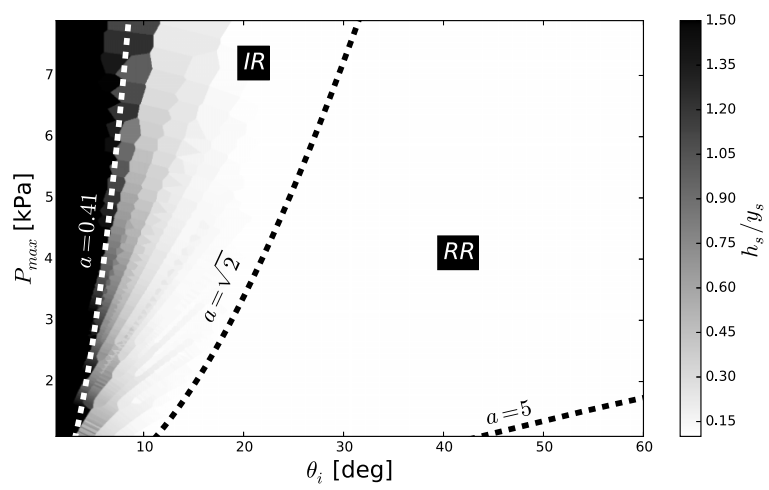

FIG. 10. Numerical normalized height $h_{s} / y_{s}$ of the Mach stem as a function of the positive peak pressure amplitude $P_{\max }$ and the incidence angle $\theta_{i}$. IR: Irregular reflection; RR: Regular reflection. 


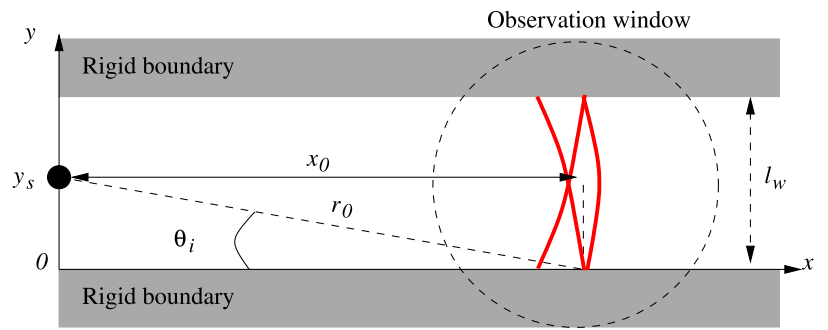

FIG. 11. Schematic representation of the geometrical setup. A spherically diverging shock wave generated by the spark source located at $\left(0, y_{s}\right)$ propagates between the two parallel boundaries and passes through the observation window whose center is located at the distance $x_{0}$ from the spark source.

considered. In this case, it would be necessary to use a three-dimensional model to take into account the spherical divergence.

\section{PROPAGATION BETWEEN PARALLEL RIGID BOUNDARIES}

When a second rigid boundary is set up at a distance $l_{w}$ from the first one [See Fig. 11], new reflections arise from this upper wall. In such a situation, two critical parameter $a_{t}$ and $a_{b}$ can be defined, where $a_{t}$ is associated with the first top reflection (from the boundary located at $y=l_{w}$ ) and $a_{b}$ to the first bottom reflection (from the boundary located at $y=0$ ). As presented in Fig. 12 which reports results of numerical distributions of the normalized acoustic pressure $p_{a} / P_{\max }$ (top figures) and schlieren measurements (bottom figures) for $l_{w}=84 \mathrm{~mm}$ and $a_{b}=0.83,0.66,0.45$, and 0.22 , the reflection patterns on the lower boundary remain the same as the ones previously presented [see Fig. 7]. The boundary located at $y=l_{w}$ gives rise to new reflections whose regimes are governed by the parameters $a_{t}=2.13,2.25,2.33$, and 2.34 as seen in Figs. 12(a)-12(d), respectively. Experimental and numerical results are in good agreement whether it is for the locations and shapes of the front and rear shocks of both incident and reflected waves, and for the Mach stem.

Adjusting the source location at equal distance from each of the two boundaries $\left(y_{s}=l_{w} / 2\right)$, the reflection patterns become symmetrical with respect to the axis $y=y_{s}$ as shown in Fig. 13 presenting results of numerical distributions of the normalized acoustic pressure $p_{a} / P_{\max }$ (top figures) and schlieren measurements (bottom figures) for three propagation distances. The gap between the two boundaries is here $l_{w}=42 \mathrm{~mm}$ and parameter $a=a_{t}=b_{t}$ is successively equal to $a=0.71,0.51$, and 0.44 in Figs. 13(a)-13(c), respectively. When the angle of incidence decreases
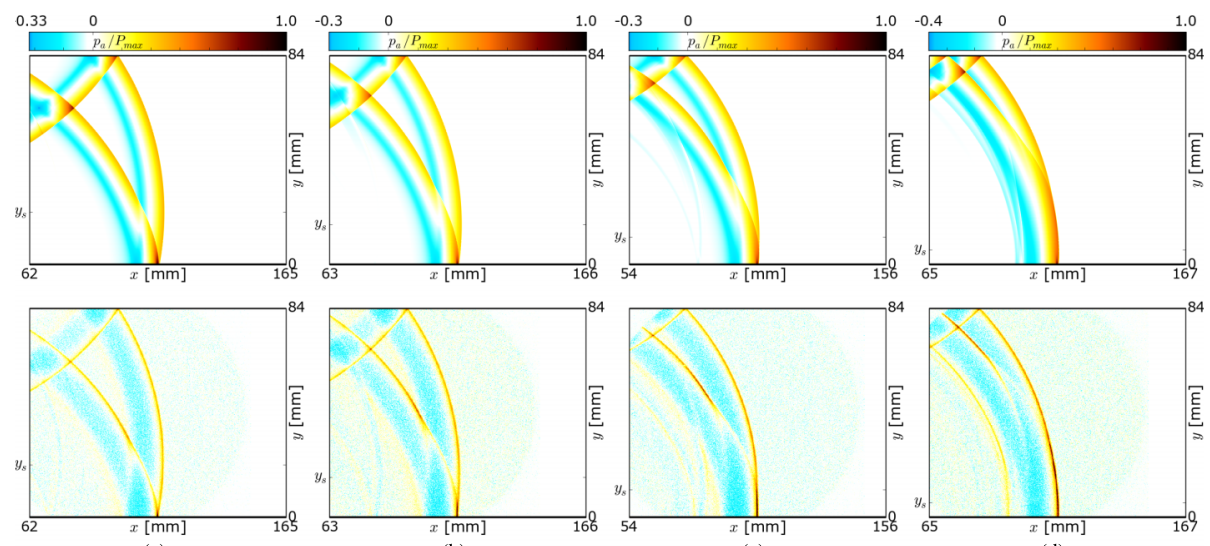

(b)

(c)

(d)

FIG. 12. Comparison of the numerical spatial distributions of acoustic pressure (top figures) and schlieren images (bottom figures) between the two parallel boundaries spaced from $l_{w}=84 \mathrm{~mm}$ for (a) $a_{b}=0.83, a_{t}=2.13$, (b) $a_{b}=0.66, a_{t}=2.25$, (c) $a_{b}=0.45, a_{t}=2.33$, and (d) $a_{b}=0.22, a_{t}=2.34$. 


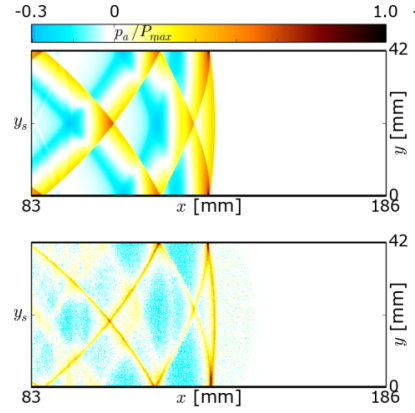

(a)

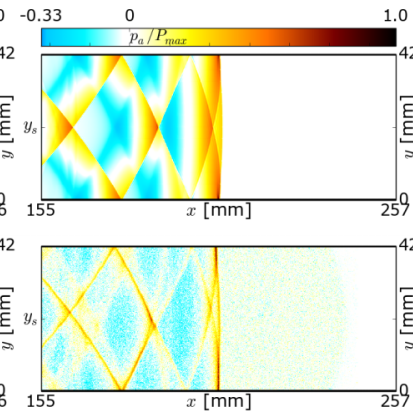

(b)

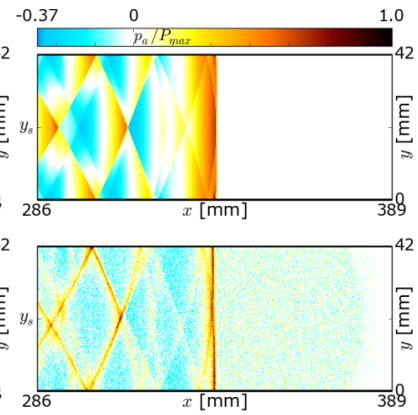

(c)

FIG. 13. Comparison of the numerical spatial distributions of acoustic pressure (top figures) and schlieren images (bottom figures) between the two parallel boundaries for $l_{w}=42 \mathrm{~mm}$ and for (a) $a=0.71$, (b) $a=0.51$, and (c) $a=0.44$.

as a function of the propagation distance, the critical parameter $a$ associated with each of the two reflections arising from each of the two boundaries decreases as well. Accordingly, the height $h_{s}$ of the two Mach stems increases. As shown in Fig. 14 which presents the comparison of the numerical distribution of acoustic pressure and schlieren measurement for $a=0.19$, when the height of the Mach stem is greater than the half-distance $l_{w} / 2$ between the two boundaries, the initial spherically diverging acoustic shock wave loses its sphericity and a plane shock wave is created between the two parallel boundaries. In both experimental and numerical results, the peak overpressure is constant along the wavefront. The merging of the two Mach stems does not involve pressure amplitude discontinuity in this case. It is worth noting that the Mach stems emerging from the reflections on the two boundaries can join as long as $2 h_{s} \geq l_{w}$. This leads to the inequality

$$
a \leq \xi \text {. }
$$

This condition corresponds to the case where the height $h_{s}$ of the Mach stem can exceed the height of the source $y_{s}$.

Fig. 14 also shows that experimental and numerical results are slightly different. Whereas successive reflections are clearly identified in the numerical results, the experiment shows a single plane shock followed by another plane shock of lower amplitude emerging from an overall diffuse field. Moreover, the duration of the overpressure following the shock front is larger in the numerical case than in the experimental one. These differences definitely originate (i) numerically, from the three-dimensional effects that were not taken into account in the model and (ii) experimentally, from the use of a two-dimensional optical method implying an averaging in the plane $(x, y)$ of the multiple reflections. These effects seem to have a significant impact on the spatial distribution of the acoustic pressure in this particular configuration (merging of the Mach stem leading to a strong maximum peak positive amplitude, narrow waveguide leading to low angle of incidence and multiple reflections).

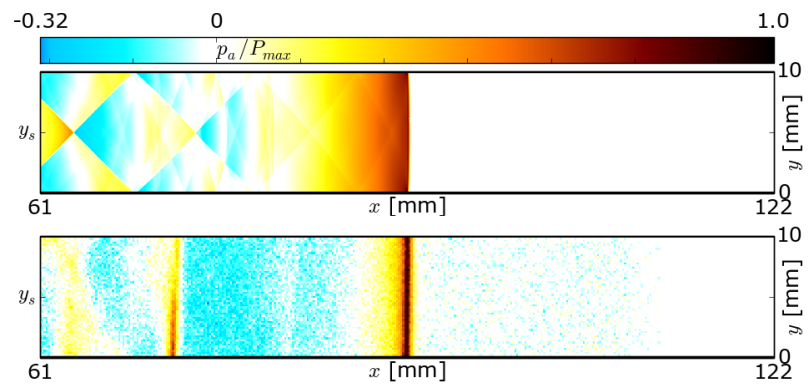

FIG. 14. Comparison of the numerical spatial distributions of acoustic pressure (top figure) and schlieren images (bottom figure) between the two parallel boundaries for $l_{w}=10 \mathrm{~mm}$ and $a=0.19$. 


\section{CONCLUSIONS}

The reflection on a rigid boundary of a spherically diverging shock wave produced by an electrical spark source is investigated experimentally and numerically. The comparison of the two-dimensional numerical spatial distributions of acoustic pressure and the schlieren measurements shows good agreement. These results show that the use of DNS based on a Navier-Stokes solver seems promising as a predictive tool to study nonlinear acoustic propagation of acoustic waves in complex geometrical configurations with rigid boundaries. Different regimes of reflection have been observed as a function of the critical parameter $a$. It has been shown that there exists a limit for which there is a transition between the regular and irregular regimes of reflection according to the results obtained by Baskar et al. ${ }^{7}$ in the case of plane acoustic shocks. It has also been shown that, when the regime of reflection is irregular, a third shock called Mach stem can appear, and that its height evolves linearly with the propagation distance. Moreover, this height is directly linked to the critical parameter $a$. When a second boundary is located parallel with the first one, the reflection patterns remain identical. When the critical parameter $a$ is lower than $\xi=0.41$, the interaction of the Mach stems arising from each of the two boundaries can lead to the formation of a plane shock wave in the waveguide.

\section{ACKNOWLEDGMENTS}

This work is supported by the french Agence Nationale de la Recherche (ANR), under Grant No. ANR-12-ASTR-0038 (project LORETA) and by the LabEx CeLyA (No. ANR-10-LABX-60). The authors would like to thank Jean-Michel Perrin for his precious help for the conception of the experimental setup. The authors are also indebted to Thomas Castelain for his numerous recommendations concerning the setup of the schlieren system, and Emmanuel Jondeau and Nathalie Grosjean for their helpful assistance concerning the optical devices. The authors are finally grateful to Petr Yuldashev for his work on the Mach-Zehnder Interferometer.

${ }^{1}$ E. Mach, “Über den Verlauf von Funkenwellen in der Ebene und im Raume,” Sitzungsbr. Akad. Wiss. Wien 78, 819-838 (1878).

${ }^{2}$ G. Ben-Dor, Shock Wave Reflection Phenomena (Springer, 1992).

${ }^{3}$ J. von Neumann, "Oblique reflection of shock," in Collected Works of John von Neumann (Pergamon Press, MacMillan, New York, 1963), Vol. VI, pp. 238-299.

${ }^{4}$ P. Colella and L. F. Henderson, “The von Neumann paradox for diffraction of a weak shock wave," J. Fluid. Mech. 212, 71-94 (1990).

${ }^{5}$ B. Birkhoff, Hydrodynamics, a Study in Logic, Fact and Similitude (Princeton University Press, 1950).

${ }^{6}$ V. W. Sparrow and P. Raspet, "A numerical method for general finite amplitude wave propagation in two dimensions and its application to spark pulses," J. Acoust. Soc. Am. 90(5), 2683-2691 (1991).

${ }^{7}$ S. Baskar, F. Coulouvrat, and R. Marchiano, "Nonlinear reflection of grazing acoustic shock waves: Unsteady transition from von Neumann to Mach to Snell-Descartes reflections," J. Fluid. Mech. 575, $27-55$ (2007).

${ }^{8}$ E. A. Zabolotskaya and V. R. Khokhlov, "Quasi-plane waves in the nonlinear acoustics of confined beams," Sov. Phys. Acoust. 15, 35-40 (1969).

${ }^{9}$ M. Brio and J. K. Hunter, "Mach reflection for the two-dimensional Burgers equation," Physica D 60, 194-207 (1992).

${ }^{10}$ R. Marchiano, F. Coulouvrat, S. Baskar, and J. L. Thomas, "Experimental evidence of deviation from mirror reflection for acoustical shock waves," Phys. Rev. E 76, 056602 (2007).

${ }^{11}$ M. Karzova, V. Khokhlova, E. Salze, S. Ollivier, and P. Blanc-Benon, "Mach stem formation in reflection and focusing of weak shock acoustic pulses," J. Acoust. Soc. Am. 137(6), EL436 (2015).

${ }^{12}$ M. Karzova, P. Yuldashev, V. Khokhlova, S. Ollivier, and P. Blanc-Benon, "Application of a Mach-Zehnder interferometer to the observation of Mach stem formation when a shock wave is reflected from a rigid surface," Bull. Russ. Acad. Sci.: Phys. 79(10), 1293 (2015).

${ }^{13}$ J. Picaut and L. Simon, "A scale model experiment for the study of sound propagation in Urban areas," Appl. Acoust. 62(3), 327-340 (2001)

${ }^{14}$ W. M. Wright, "Propagation in air of N waves produced by sparks," J. Acoust. Soc. Am. 73(6), 1948-1955 (1983).

${ }^{15}$ C. Ayrault, P. Béquin, and S. Baudin, "Characteristics of a spark discharge as an adjustable acoustic source for scale model measurements," in Proceedings of the Acoustics 2012, Nantes, France (Société Française d'Acoustique, 2012), pp. 1112-1115.

${ }^{16}$ G. S. Settles, Schlieren and Shadowgraph Techniques (Springer, 2001).

${ }^{17}$ B. André, T. Castelain, and C. Bailly, "Shock-tracking procedure for studying screech-induced oscillations," AIAA J. 49(7), (2011).

${ }^{18}$ M. Karzova, P. Yuldashev, V. Khokhlova, S. Ollivier, E. Salze, and P. Blanc-Benon, "Characterization of spark-generated $N$-waves in air using an optical schlieren method," J. Acoust. Soc. Am. 137(6), 3244-3252 (2015). 
${ }^{19}$ W. E. Lorenson and H. E. Cline, "Marching cubes: A high resolution 3D surface construction algorithm,” ACM SIGGRAPH Comput. Graphics 21(4), 163-170 (1987).

${ }^{20}$ P. Yuldashev, M. Karzova, V. Khoklova, S. Ollivier, and P. Blanc-Benon, "Mach-Zehnder interferometry method for acoustic shock wave measurements in air and broadband calibration microphones," J. Acoust. Soc. Am. 137(6), 3314-3324 (2015).

${ }^{21}$ G. Smeets, "Laser interference microphone for ultrasonics and nonlinear acoustics," J. Acoust. Soc. Am. 61(3), 872-875 (1977).

22 J. W. Reed, "Atmospheric attenuation of explosion waves," J. Acoust. Soc. Am. 61, 39-47 (1977).

${ }^{23}$ P. Yuldashev, S. Ollivier, M. Averiyanov, O. Sapozhnikov, V. Khoklova, and P. Blanc-Benon, "Nonlinear propagation of spark-generated $N$-waves in air: Modeling and measurements using acoustical and optical methods," J. Acoust. Soc. Am. 128(6), 3321-3333 (2010).

${ }^{24}$ E. Salze, P. Yuldashev, S. Ollivier, V. Khokhlova, and P. Blanc-Benon, "Laboratory-scale experiments to study nonlinear $N$-wave distortion by thermal turbulence," J. Acoust. Soc. Am. 136(2), 556-566 (2014).

${ }^{25}$ O. Marsden, C. Bogey, and C. Bailly, "A study of infrasound propagation based on high-order finite difference solutions of the Navier-Stokes equations," J. Acoust. Soc. Am. 135(3), 1083-1095 (2014).

${ }^{26}$ C. Bogey and C. Bailly, "A family of low dispersive and low dissipative explicit schemes for noise computation," J. Comput. Phys. 194(1), 194-214 (2004).

${ }^{27}$ C. Bogey, N. De Cacqueray, and C. Bailly, "A shock-capturing methodology based on adaptative spatial filtering for high-order non-linear computations," J. Comput. Phys. 228, 1447-1465 (2009).

${ }^{28}$ C. de Groot-Hedlin, "Finite-difference time-domain synthesis of infrasound propagation though an absorbing atmosphere," J. Acoust. Soc. Am. 124(3), 1430-1441 (2008).

${ }^{29}$ P. Yuldashev, M. Averiyanov, V. Khokhlova, S. Ollivier, and P. Blanc-Benon, "Nonlinear spherically divergent shock waves propagating in a relaxing medium," Acoust. Phys. 54(1), 32-41 (2008). 